\title{
Writing The Literature Review
}

\author{
Joel Rey U. Acob ${ }^{1,}$ Heru Santoso Wahito Nugroho ${ }^{2}$ \\ ${ }^{1}$ Faculty of Nursing, Visayas State University, Philippines (joel.acob@vsu.edu.ph) \\ ${ }^{2}$ Health Polytechnic of Surabaya, Indonesia (heruswn@gmail.com)
}

Submitted: December 2, 2018 -Revised: January 18, 2019 -Accepted: January 21, 2019 -Published: January 31, 2019

\begin{abstract}
In the development of scientific investigation, traditionally, a researcher identifies prevailing issues as the focus of study. Following the institutional format gives way to decide within the box since conventional approach of accomplishing research are utilized. This article guides the neophyte researchers to alter the "customary" dealings of analyzing phenomenon under study without deviating the protocol.
\end{abstract}

Keywords: Literature, Literature review, Integrative review, Systematic review

\section{PURPOSES OF LITERATURE REVIEW}

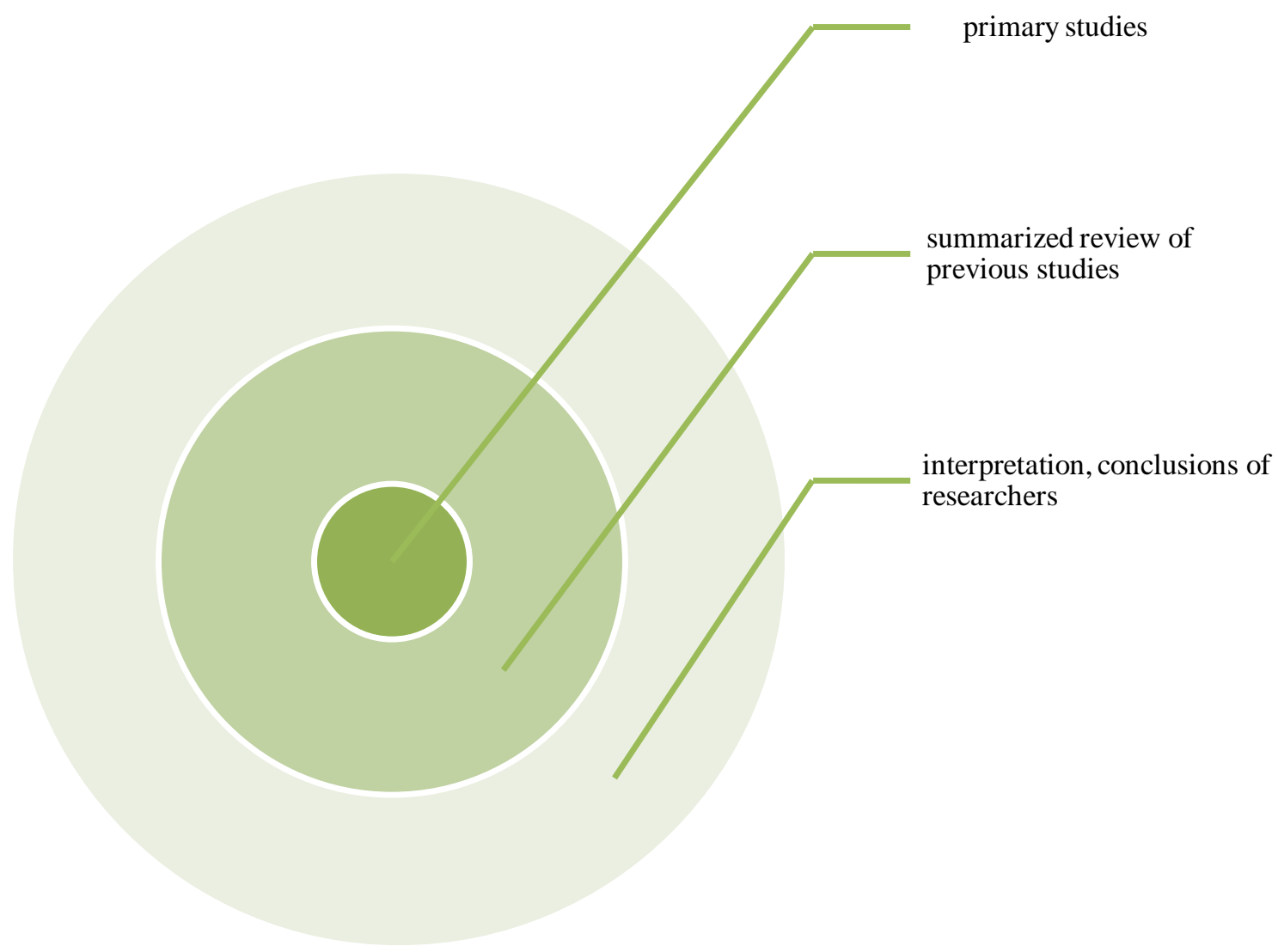

Figure 1. Layers of Literature Review 
Straightly speaking, the very core of literature review is to identify the extent of problem under investigation. Ideally, researchers engaged in such endeavor due to predicament related to one's existence. Problems identified needs specific refinement as one of the characters of research opportunities. Questions have to be developed scientifically since the process demands systematic exploration. Review of related literature (RRL) orients scholars to both what is known and not about the area of inquiry. RRL is a collection of study findings from periods of recognition until the moment researchers decided to conduct similar examination. Though informations are huge and perhaps related that of other studies, but considering these were performed in different settings, samples were taken from diversed atmosphere- that findings are not absolutely the same. RRL ables to determine gaps and any inconsistencies in a body of knowledge.

Further, it [litertures] gives meaning and relevance in discovering theoretical and conceptual frameworks relevant to research problems. Theoretical frameworks are required for qualitative research designs where exploration and understanding of human nature are focused. In qualitative designs, researchers have to discover participants' experiences ${ }^{1}$ as major source of significant themes. On one hand, conceptual framework embodies expected output(s) as researchers identify relationship [if any] among variables under study. Development of hypothesis (es) to be tested is imperative in quantitative designs.

Another purpose of RRL is to assist scholars especially during interpretation of results. When findings are recognized, investigators create conjectures. Normally, they [researchers] utilized the synthesized literatures to expound implications. It either support or has a contrarian viewpoint of the previous findings. Researchers will have new ways to read between lines as they describe relationships of each work to others under consideration.

Researchers are also called to advance search areas of prior scholarship to avert duplication of efforts. To this purpose, RRL is able to resolve conflict among seemingly contradictory previous studies. As mentioned, not all similar findings are alike; not replicated, just comparable. With new findings, one can locate his/her own research within the context of exisiting literature.

Literature review surveys books, scholarly articles, and any other sources germane to a particular subject, or theory, and by so doing, grants of narrative, summary and critical evaluation of these works in relation to the research problem under investigation. The most basic among the three (3) are primary studies that researchers conduct and publish. The dead-end of any research activity is publication of findings, which is to communicate answers based on statements of opportunities. Primary studies refer to fundamental research result whereby addressing specific problems. Literature summarizes findings and offers new angles of interpretation of phenomenon being studied. Reviews sometimes extend the common pattern of analysis even beyond primary studies. Further, the third layers of literature are those that are shared informally that become part of the lore of the field. Individual perceptions of the researcher, developed conclusions based from study findings belong to this stratum.

\section{TYPES OF LITERATURE REVIEW}

The types of literature review are:

1. Argumentative review. This aspires to widen a body of literature that establishes a contrarian viewpoint. Researcher intends to create another version of what was investigated in the past. This type of review totally challenged earlier discussions; hence, researcher expands the horizon of the current study. Additionally, argumentative review scrutinizes selectively in order to maintain or refute a case, deeply imbedded theory, or supposition, and philosophical problem already instituted in the literature.

2. Historical review. It intends to trace origin and its evolution with the scholarship of discipline. This type of review starts from the very beginning of an issue, concept of an emerging theory ${ }^{3}$ and follow a line of investigation. Historical review is used to demonstrate familiarity with the state-of-the-art growth and categorize the likely information for future research.

3. Methodological review. Reviews do not always focus on what [findings], but how they came about saying what they say [method of analysis]. Ethical issues are highlighted so wider awareness and consideration for the study is uplifted. The approach provides framework to better value a wide diversity of comprehension from quantitiative to qualitative integration. Practical documents are outputs of conceptual framework, while sampling, data collection, and analyses are believed as necessary too.

4. Theoretical review. This form is utilized to facilitate and establish a lack of appropriate theories or disclose that contemporary theories are inadequate to explain emerging research problems. A theoretical concept or the whole framework may be used as the unit of analysis. Researchers employ theoretical review to explain 
relationship between assumptions, and as to what degree the existing theories have been inspected, and to increase new hypothesis to be tested.

5. Systematic review. Typically, it focuses on a very specific emprical question, often posed in a cause-andeffect form, such as "to what extent does A contribute to B?" The question is singled out from a clearly formulated idea. It overviews current evidence pertinent to which the question was taken into account. It also applies pre-specified and standardized methods to classify and critically appraise relevant research, collects, and reports data from the studies that are contained in the review.

6. Integrative review. This is the most common form of review in social sciences. Normally, the analysis includes all studies that concentrate on related and contrasting to identical hypothesis or research problems and opportunities. It further analyzes representative texts and synthesizes literatures on a topic in an integared way such that new shape of perspective and frameworks are generated.

\section{DEVELOPMENT OF LITERATURE REVIEW}

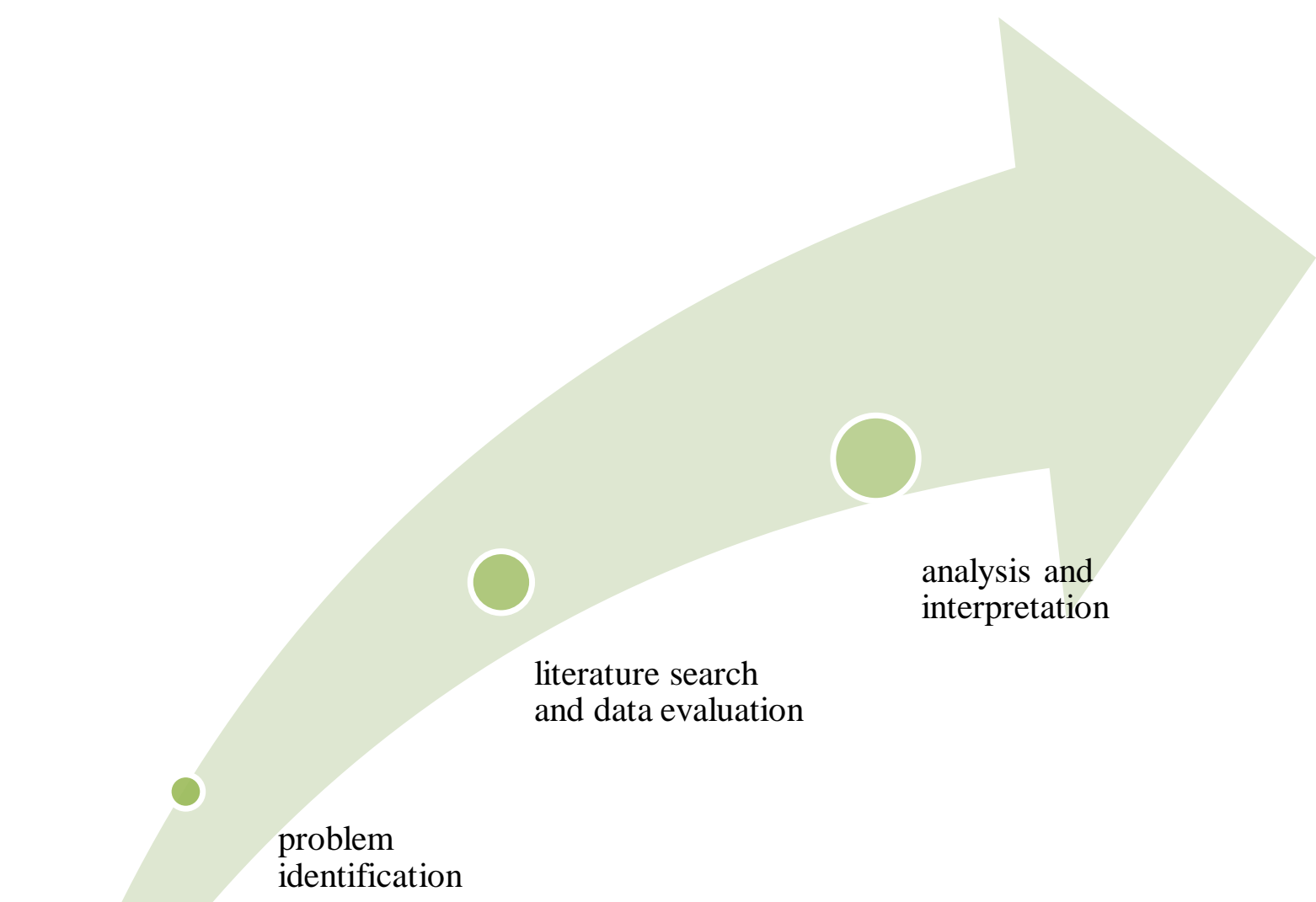

Figure 2. Steps in developing literature review

Constructing literature review also follows the configure of typical research. Problem identification drives the entire process that researchers should establish prior to recognizing potential sources of information. Search engines are commonly utilized to retrieve significant researches as unit of analysis. Key words are then employed 
so studies can be explored. Investigator should be knowleagable regarding search engine to include technique utilization for extraction of major studies. Other researchers operate selection criteria in naming salient researches. Suppose you wanted to read about the changing roles of nurse managers ${ }^{1}$. Keywords such as role, development, nurse managers, health care, changing care practice are some of the many key-in words in the search engine. Year of publication, peer-reviewed, scholarly journal and the origin that of study are also added for a conservative output.

Evaluation of data is done when researcher has reached its maximum reads and/or no studies become qualified as far as the criteria are concerned.

Researchers should have decisiveness prior to literature review writing. Considerations like the types of sources to be reviewed must be established to save time and resources. The intention of the conducting the review should be cleared prior to engine search so only those that bears weight to topic of interest is included especially in the abstract appraisal ${ }^{4}$. Evaluating the source where the information is lifted should also be reliable. Today, the issue of data accuracy is at stake especially at the high edvent of technology. Some researchers rely on online information obtained from the internet sources, however there is poor validation and cross-checking scheme to assure that such data are true and correct. Teachers must ensure that only those vital findings undergoing peerreview and evaluation process are ones communicated.

\section{WRITING LITERATURE REVIEW}

Conventionally, literature development comes after identification of problem's section. For neophytes, literature reviews (LR) are only utilized to strengthen claim of such endeavor or as prop up during the interpretation of findings. However, LR guides researcher to discover what was known and not at the topic of interest. In writing literatures, the following points are given emphasis:

1. Use evidence. Confirmation of studies included for review demonstrates genuity of the study in itself. This expresses further the researcher's advocacy in the utility of published research (es) in a reputable journal.

2. Be selective. Identifying research outputs is not a shopping-event. Hence, researchers should become choosy during the selection process following the criteria established beforehand.

3. Use quotes sparingly. Scientific manuscripts are seldom made of proverbial texts. Though researchers sometimes bank on "sayings" as springboard to elaborate or create analogy commonly observed on qualitative studies.

4. Summarize and sythesize. Research has the attribute of replication and duplicity. In literature review, outputs are comprised synopsis of examined and analyzed studies.

5. Use caution when paraphrasing. Make sure that content and meaning of the original studies is intact amidst rephrasing.

\section{REFERENCES}

1. Acob JRU. How to Write Abstracts for Health Research Articles?. Health Notions. 2017;1(3):300. https://doi.org/10.33846/hn.v1i3.289.

2. Acob JRU, Martiningsih W. 2018. Role Development of Nurse Managers in Changing Health Care Practice. Jurnal Ners dan Kebidanan. 2018;5(1). DOI: 10.26699/jnk.v5i1.ART.p066-068.

3. Acob JRU. Caring as Unending Expression of Nursing (CUEN): A Theory of Nursing. Malaysian Journal of Nursing. 2018;10(2):52-56. doi:10.31674/mjn.2018.v10i02.006

4. Acob JRU. Lived Experiences of the Abused Elderly. Public Health of Indonesia. 2018;4(1):1-8. http://stikbar.org/ycabpublisher/index.php/PHI/index 